\title{
FUNDAMENTOS DE LA CALIDAD DE SERVICIO, EL MODELO SERVQUAL
}

\section{FUNDAMENTALS OF QUALITY OF SERVICE, THE SERVQUAL MODEL}

\author{
Miguel A. Bustamante U. ${ }^{12},{ }^{*}$ Elsie Zerda, ${ }^{2}$ Francisco Obando, ${ }^{3}$ Michelle Tello, ${ }^{4}$ \\ 1 Facultad de Economía y negocios, Universidad de Talca, Dos Norte 685, 3465548 Talca, Chile. E-mail: mabu@utalca.cl \\ Tel.: +56712200 321. Fax: +56712200457. \\ 2 Facultad de Especialidades Empresariales, Universidad Católica de Santiago de Guayaquil, 090615 Guayaquil, Ecuador. e-mail: elsie.zerda @cu.ucsg.edu.ec \\ 3 Facultad de Ciencias Médicas, Universidad Católica Santiago de Guayaquil, Guayaquil, Ecuador. Av. Carlos Julio Arosemena Km. 1¹⁄2 vía Daule, Guayaquil, Ecuador. E-mail: francisco. \\ obado@cu.ucsg.edu.ec \\ 4 Facultad de Ciencias Económicas y Administrativas, Universidad Católica de Santiago de Guayaquil, 090615 Guayaquil, Ecuador. \\ e-mail: michelle.tello @cu.ucsg.edu.ec
}

Antecedentes: Los modelos teóricos de calidad que involucran conceptos de expectativa y percepción son unos de los más referidos en las publicaciones científicas. En d iferentes países se ha buscado determinar si este modelo es una medida de calidad válida en el contexto de sus instituciones de salud.

Objetivo: Sintetizar la evidencia científica relacionada a las adaptaciones y usos del modelo Servqual en el contexto hospitalario.

Metodología: Se realizó una revisión sistémica exploratoria mediante la búsqueda de artículos de investigación en bases de datos PubMed, Sciencedirect y Scopus en un periodo de 2013-2018.

Resultados: Se recopilaron un total de 62 publicaciones, la mayoría de artículos son producidos en la región asiática. Los autores encontrados suponen a la escala Servqual como un instrumento válido en el contexto de su estudio.

Conclusión: La escala aún después de más de 30 años desde su origen sigue siendo extensamente aplicado en el contexto sanitario. Las dimensiones iniciales planteadas por el autor no siempre se mantienen en todos los contextos hospitalarios, investigadores deberían llevar a cabo pruebas de validez para confirmar si los conceptos de empatía, seguridad, fiabilidad, capacidad de respuesta y elementos tangibles, persisten y se ratifican en la muestra de su estudio.

Palabras clave: Servicios de salud, calidad de atención de salud, modelo de brechas, expectativas, percepciones.
Background: The theoretical models of quality that involve concepts of expectation and perception are some of the most referred in scientific publications. In different countries, it has been sought to determine if this model is a valid quality measure in the context of its health institutions.

Objective: Synthesize the scientific evidence related to the adaptations and uses of the Servqual model in the hospital context.

Method: An exploratory systemic review was carried out by searching for research articles in PubMed, Sciencedirect and Scopus databases in a period of 2013 - 2018.

Results: A total of 62 publications were compiled, most articles are produced in the Asian region. The authors found assume the Servqual scale as a valid instrument in the context of its study.

Conclusion: The scale even after more than 30 years from its origin is still widely applied in the health context. The initial dimensions proposed by the author are not always maintained in all hospital contexts, researchers should carry out validity tests to confirm whether the concepts of empathy, security, reliability, responsiveness and tangible elements persist and are ratified in the sample of your study.

Keywords: Health services, quality of health care, gap model, expectations, perceptions. 


\section{Introducción}

En las páginas siguientes se presenta una síntesis teórica del concepto de calidad, calidad de servicio conceptos relacionados, además de algunas opciones metodológicas que permiten su medición.

\section{El concepto de calidad}

La calidad se puede definir como un concepto relativo (Schroeder, 1992) al uso esperado y al cliente debido a que difiere según sea el producto, servicio y tipo de empresa en que se opere y sin duda alguna, respecto del tipo de cliente que se trate. Ésta posee diferentes enfoques (Aguayo, 1993; Donabedian, 1966; Horovitz, 1991; Schroeder, 1992), pero una definición más adecuada hace mención a que la calidad es la totalidad de los rasgos y características de un producto o servicio que se sustenta en su habilidad para satisfacer las necesidades y expectativas del cliente, y cumplir con las especificaciones con la que fue diseñado (Aguayo, 1993), es decir, calidad corresponde al conjunto de características y atributos de un producto o servicio respecto de las necesidades y expectativas del comprador o cliente, considerando el precio que se está dispuesto a pagar (Horovitz, 1991).

Por otra parte, se ha definido la calidad como una adecuación al uso, lo que significa que el producto o servicio satisface las necesidades de un cliente, es decir, el producto, se adapta adecuadamente al uso que espera un determinado cliente, de acuerdo con las necesidades del cliente, el producto y la forma cómo este se adapta al uso del cliente (Schroeder, 1992). Adicionalmente, Aguayo (1993), señala que la calidad debe ser considerada desde el punto de vista del consumidor, en tanto la calidad es todo aquello que realza el valor de un servicio o producto, por supuesto, en opinión de un cliente determinado. Horovitz (1991) define, desde su particular perspectiva, que la calidad es el grado de excelencia que la empresa ha escogido alcanzar para satisfacer a su mejor clientela, representando al mismo tiempo, la medida en que se logra dicha calidad.

En lo conceptual, el enfoque teórico más conocido de la calidad es el desarrollado por Donabedian (1966) quien reconoció las deficiencias en los diferentes tipos de evaluación de la calidad en el contexto salubrista, definiendo la calidad en la atención médica como un concepto multidimensional, bajo cuyo esquema, se reconoce que la percepción del paciente es un punto clave para la determinación de la calidad del servicio otorgado en cualquier institución médica (Chasin y Galvi, 1998). De hecho, una de las definiciones más concretas de las presentadas, indica que una atención médica de calidad dice relación con un tipo de atención que maximiza el bienestar del paciente, contemplando en ello un proceso de atención que aborda en todas sus partes las dimensiones funcional y técnicas más relevantes y significativas de la calidad (Galván, Moctezuma, Dolci y López, 2016).

En particular, en ambientes hospitalarios, la calidad técnica refiere a la precisión de los análisis y técnicas médicas empleadas en el diagnóstico; mientras que la calidad funcional refiere a la forma en que se entregan dichos servicios (Lam, 1997). En consecuencia, la calidad funcional en los servicios de salud es crucial, ya que su evaluación permite la mejora del servicio basada en los sentimientos, expectativas y observancia de los pacientes (Wysong y Driver, 2009).

\section{El concepto de servicio}

El concepto de servicio dice relación con acciones, procesos y ejecuciones intangibles que comprenden hechos y acciones que se realizan para y con los clientes o usuarios (Zeithaml y Bitner, 2002). Todo lo cual deriva en la necesaria capacidad de percepción que los clientes tienen o puedan llegar a tener de los servicios propiciados, considerando en ello una provisión de calidad así como del grado en que el prestador logre que hacer sentir satisfechos a sus clientes, sea en términos del producto - servicio particular como respecto de la experiencia general de satisfacción experimentada (Zeithaml y Bitner, 2002).

Un servicio se define como el conjunto de prestaciones anexas que acompañan a la prestación principal, la cual puede ser un producto tangible. En términos generales, los servicios son acciones, procesos y ejecuciones, por lo tanto las empresas y organizaciones deben prestar atención a los servicios que están entregando, pues en ellos radica la diferenciación que marcará la Satisfacción Usuaria, sobre la base de cuatro características consideradas básicas (Stanton,1966).

\section{El concepto de satisfacción}

El concepto de satisfacción reporta a la percepción íntima que experimenta el cliente o usuario y a la evaluación que éste realiza del respecto del servicio al cual se enfrenta, sea en términos de si éste respondió a sus necesidades o bien si la entrega le permite alcanzar y superar sus propias expectativas (Zeithaml y Bitner, 2002), en consecuencia, la satisfacción es un juicio acerca de los rasgos que caracterizan un producto o servicio, mismo que es definido o determinado por el cliente y que, por medio del cual, se logra responder luego de alcanzar un cierto grado de saciedad, que se sintetiza en un grado o nivel placentero de recompensa auto percibida por el propio cliente o usuario (Oliver, 1999; 1993; 1981; 1980; 1977).

En este concepto se pueden encontrar sensaciones de agrado o decepción, según las expectativas del cliente (Kotler, 1966). Al hablar de satisfacción , nos referimos a un concepto esencialmente subjetivo, pues a los clientes y consumidores les interesa obtener beneficios que a su vez se traducen en satisfacción, pero esta varia respecto del sujeto que adquiere el servicio.

Como la satisfacción es medida mediante el cumplimiento de las expectativas, es necesario definir tal concepto en términos del servició esperado por el consumidor (Oliva,2004). Al respecto, según Zeithalm y Bitner (2001), las expectativas tienen dos componentes fundamentales. El primero se denomina "Servicio deseado", mismo que se define como el nivel de servicio que el cliente espera recibir. El segundo se denomina "Servicio adecuado" el cual se corresponde con el servicio mínimo tolerable que el consumidor está dispuesto a aceptar.

\section{Concepto de percepción}

Sobre la base del modelo conceptual que se presenta, el concepto de percepción resulta vital. En principio se trata de un proceso mediante el cual un individuo selecciona, organiza e interpreta la información que recibe para crear una imagen inteligible del mundo 
(Berelsonn y Steiner,1964) y en segundo lugar, se trata que las percepciones siempre se han de considerar en relación con las expectativas, además identificar y determinar cómo los clientes perciben los servicios en términos de su calidad y el grado de satisfacción que experimentan en el proceso (Zeithaml y Bitner,2001).

\section{Calidad de servicio versus satisfacción}

Las relaciones entre la calidad de servicio y la satisfacción del consumidor o usuaria han sido extensamente discutidas, en especial por Parasuraman et al. (1988) y Cronin y Baker (1994). Parte de la literatura sugiere que la calidad de servicio y la satisfacción del consumidor son conceptos interdependientes, constructos que comparten una relación cercana (Cronin \& Taylor, 1992).

La satisfacción usuaria deriva del cumplimiento con una demanda y se corresponde con un sentimiento de saciedad (Oliver, 1993) y con un estado psicológico de satisfacción Oliver (1981) sin embargo, la calidad de servicio es el fundamento de la satisfacción expresada por el consumidor (Cronin \& Baker, 1994). Parasuraman et al. (1985) y se manifiesta en un juicio global placentero.

Existe evidencia teórica y empírica que la calidad de servicio sostiene la satisfacción y se movilizan conjuntamente pero no son constructos equivalentes (Cronin y Baker, 1994), es decir, están relacionados y dan pie a una percepción de la calidad sostenible (Parasuraman et al., 1985).

\section{Metodología}

Se realizó una revisión sistemática y exploratoria (Hernández, Fernández, Baptista, 2016) con el fin de describir el conocimiento actual acumulado respecto al tema. En este tipo de análisis se puede incluir estudios con diferentes enfoques de investigación cualitativas y cuantitativas (Mata, Reynoso, Salazar, 2006), obtener diferentes medidas de impacto (Mahía, 2002) y además el criterio de inclusión predominante es la relevancia de los manuscritos (Manchado, Tamames, López, Mohedano y Veiga de Cabo, 2009).

El análisis requirió la consecución de las siguientes fases: (1) determinación de los criterios de búsqueda; (2) definición de criterios de inclusión y exclusión; (3) elección de las estrategias y plataformas de búsqueda; (4) esquematización de los artículos encontrados; (5) elección y clasificación de artículos; (6) evaluación y análisis de datos (Ruiz, 1998).

La determinación de los criterios de búsqueda (Cabero, 2002; 2004) se presenta en la Tabla 1. Las plataformas para la búsqueda fueron PubMed, Sciencedirect y Scopus (Cubero, 2005)), en los cuales los caracteres usados fueron en su mayoría en inglés y otros en español (Marqués, 2008).

Se utilizó una metodología de revisión sistemática de literatura (RSL) que permite compilar, resumir, criticar y sintetizar fundamentos teóricos de investigaciones disponibles en fuentes abiertas sobre el tema abordado (Mata, Reynoso, Salazar, 2006). Se generaron tablas de síntesis destinadas a facilitar el análisis de los datos relevantes, empíricos o teóricos (Mahía, 2002), que se consideraron necesarios para atender los objetivos del presente documento teórico (Manchado, Tamames, López, Mohedano y Veiga de Cabo, 2009). Los fundamentos de esta metodología se enfocan en el conocimiento del estado del arte, la identificación de tendencias de investigación y, asimismo, la identificación de puntos de controversia (Suter, 2013; Jadika, Khoo, \& Na, 2011).

Tabla 1 Criterios para la búsqueda de artículos

$\begin{array}{ll}\text { Periodo de estudio } & \text { Últimos cinco años }(2013-2018) \\ \text { Idioma } & \text { Inglés y Castellano } \\ \text { Límites conceptuales } & \begin{array}{l}\text { Estudios basados en el modelo percepción - expectativa. Se excluyen estudios de calidad de servicio de } \\ \text { salud que utilicen otros modelos de medición de percepción similares como Servperf, Servhos, entre } \\ \text { otras adaptaciones cuyo enfoque haya perdido el concepto básico de gap o brecha de Parasurman } \\ \text { et al. (1985) }\end{array}\end{array}$

Se procedió a la preparación de tablas de revisión e integración de contenidos (Mahía, 2002) para facilitar la correspondiente narrativa y sistematización buscada, implicando esto las fases de obtención de bases de datos especializadas, clasificación de los hallazgos a fin de determinar patrones comunes y puntos de divergencia (Pautasso, 2013) $\mathrm{y}$, finalmente, definir las revisiones pertinentes de carácter conceptual y aplicado sobre la base de datos estadísticos (Hernández, Fernández, Baptista, 2016).

Además, se recurrió a la sistematización mediante una plantilla de registro de tres bases de datos especializadas (Mahía, 2002), a decir: Scopus, Web of Science y Publish or Perish (Google Scholar), de las cuales se recogieron manuscritos y documentos académicos pertinentes al campo estudiado, en este caso asociado a los temas de Calidad, Servicio, Calidad de Servicio y Percepción, lo que incluyó revisiones, comparaciones y análisis de frecuencias específicas en aspectos tales como: autores, años, citaciones y fuentes, entre otros (Suter, 2013; Jadika, Khoo, \& Na, 2011).

Finalmente, se procedió a la selección de documentos pertinentes y evaluación de la calidad documentaria a través del estudio de los abstracts de las publicaciones, verificando además los indicadores de impacto (Mahía, 2002) de SCImago Journal \& Country Rank. 


\section{Resultados}

A continuación se presentan los hallazgos conceptuales obtenidos de las diversas fuentes analizadas. Se sintetizan los hallazgos mediante modelos esquemáticos $\mathrm{y}$ tablas de registro debidamente referenciados tanto de la evolución conceptual como de las diversas aplicaciones y estudios empíricos realizados.

\section{Artículos analizados}

Se obtuvo una primera cifra potencial de publicaciones tras la búsqueda inicial en las plataformas de bases de datos (Mahía, 2002). Posterior a ello, el primer descarte fue dado por: (1) aplicaciones en otros contextos, (2) evaluaciones de calidad técnica, es decir, sin el enfoque de percepción, (3) evaluaciones solo de percepción sin el enfoque de expectativa.

El resto de artículos cuyo contenido manifestaba el concepto de Servqual o modelos de satisfacción con el enfoque de brechas, fueron obtenidos y organizados. La Figura 1 presenta un diagrama de flujo que resume el proceso de selección ejecutado.

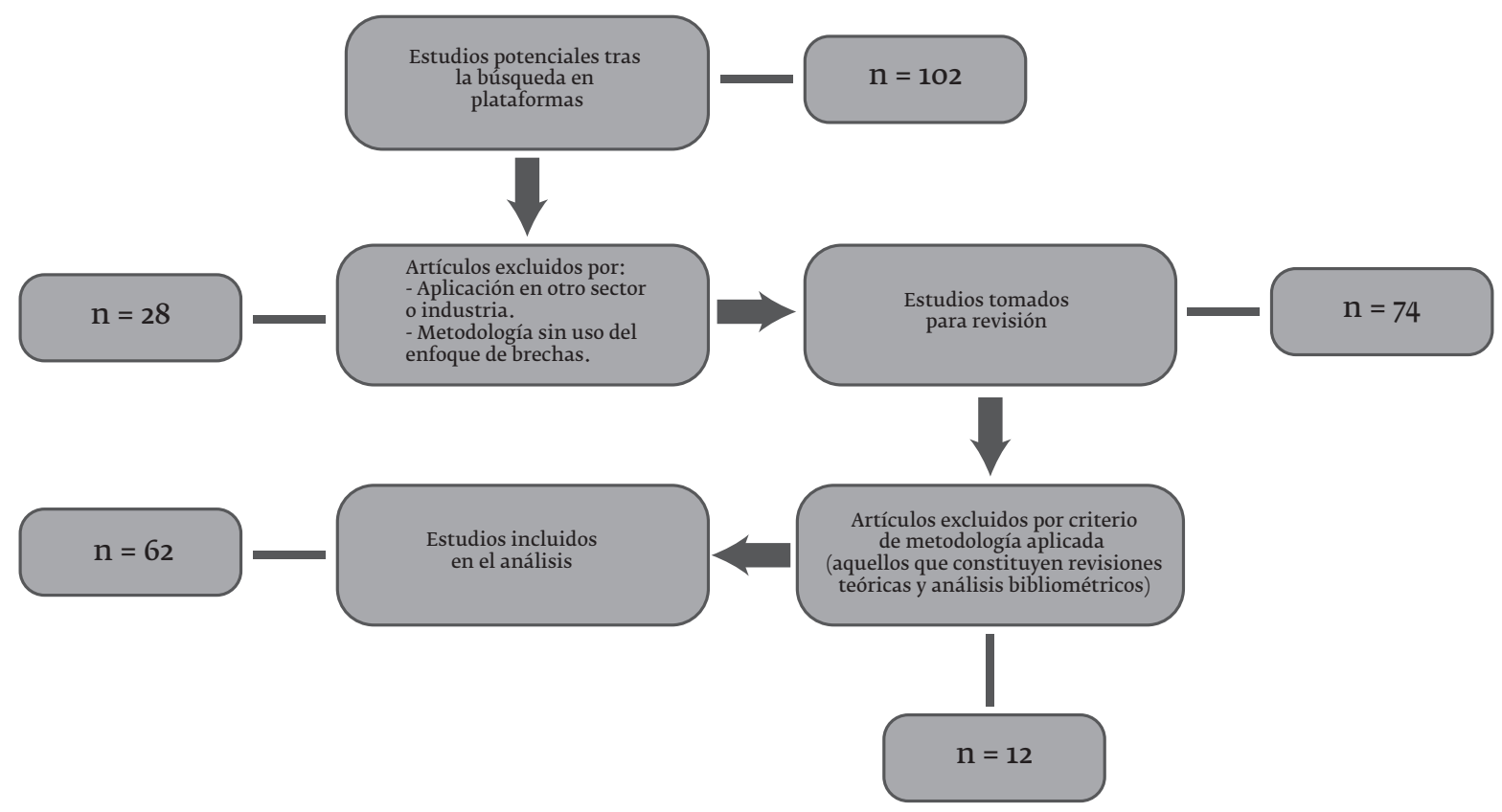

Figura 1: Diagrama de flujo de proceso de selección de publicaciones

Se procedió además, a la revisión más exhaustiva de cada uno y a una última eliminación de estudios fue dada por el criterio de la metodología empleada.

Aquellas publicaciones puramente de revisión de literatura, bibliográfica y teórica fueron sintetizados para extraer sus líneas fundamentales. El número final de artículos incluidos en el análisis fue $n=62$
Medidas bibliométricas

De las 62 publicaciones seleccionadas para el análisis el mayor porcentaje (24\%) fue publicado en el año 2018. En general, el número de publicaciones respecto a la aplicación de Servqual en diferentes contextos hospitalarios va en aumento, a excepción del año 2014, en el cual se registró un número menor de estudios respecto a su año precedente (Tabla 2).

Tabla 2 Número de artículos publicados por año

\begin{tabular}{lcccccc}
\multicolumn{7}{c}{ Tabla 2 Número de artículos publicados por año } \\
\hline Año & 2013 & 2014 & 2015 & 2016 & 2017 & 2018 \\
$\begin{array}{l}\text { Número de } \\
\text { artículos }\end{array}$ & 10 & 6 & 7 & 10 & 14 & 15 \\
\hline
\end{tabular}

Por otro lado, los países asiáticos son los que más aportan a la producción de la temática, más de la mitad de los artículos seleccionados $(58 \%)$ para el análisis provienen de esta región. En la región, los países con más publicaciones corresponden a Irán e India, con 8 estudios cada uno. Y, el resto de países representantes en el continente son China $(n=2)$, Pakistán $(n=5)$, Arabia Saudita $(n=3)$, Tailandia $(n=2)$, Turquía $(n=5)$ y Malasia $(n=2)$.

Latinoamérica constituyó la segunda región con mayor número de artículos, en la cual se registran publicaciones de Brasil $(\mathrm{n}=8)$, Colombia $(\mathrm{n}=3)$ y Perú $(\mathrm{n}=2)$. A las Américas, le sigue Europa, donde destaca Rumania y Portugal con dos publicaciones cada uno, y también se encontró estudios realizados por autores de Portugal, Croacia, España, Bosnia y Albania.

Por último, Nigeria es el país con más producción científica del tema en la región africana, y se registraron estudios adicionales en la región de países como Ghana, Tanzania y Etiopía (Tabla 3). 
Tabla 3 Número de artículos publicados por región

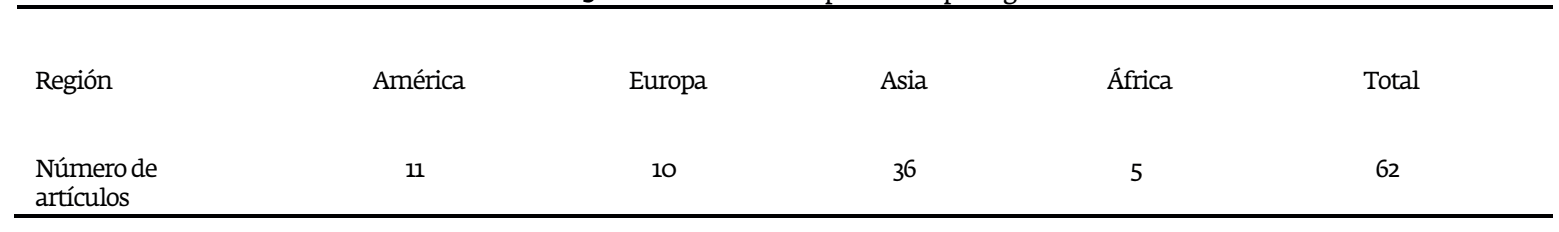

Entre otros datos relevantes a mencionar es que entre los autores quienes han producido más de un artículo dentro de la temática de Servqual en el contexto hospitalario en los últimos cinco años se encuentran Javed et al. (2018a, 2018b) y Purcărea (Purcărea et al., 2013; Gheorghe, Gheorghe, \& Purcărea, 2018). Un importante número de publicaciones se registraron bajo las editoriales de Emerald Insight $(\mathrm{n}=7)$, Elsevier $(n=6)$ y Taylor\&Francis $(n=6)$.

\section{Síntesis bibliográfica}

Se llevó a cabo el conteo, revisión exhaustiva y análisis delos registros, el cual consistió en la esquematización de los principales métodos, muestras y resultados obtenidos en los trabajos recopilados.

La Tabla 4 presenta un resumen de la literatura revisada ordenada en forma cronológica. En particular, los autores que han aplicado el Servqual como medida de calidad en el contexto hospitalario, suponen a la satisfacción, percepciones y expectativas como medidas fiables para la evaluación de la calidad.
En consecuencia, no todos los estudios aplican prueba de validez y fiabilidad para verificar la consistencia del instrumento.

En particular, del número de publicaciones consideradas para el análisis, un total de ocho autores modelan y verifican la estructura factorial de la escala mediante análisis factorial exploratorio (AFE), confirmatorio (AFC) y modelamiento estructural (SEM), de los cuales algunos confirman la estructura de las cinco dimensiones del Servqual (Purcărea et al., 2013; Yousapronpaiboon \& Johnson, 2013; Jandavath \& Byram, 2016; Cengiz \& Fidan, 2017).

Sin embargo, otros estudios condensan los reactivos en solo cuatro dimensiones (Abuosi \& Atinga, 2013; Musa et al., 2018) sin perjuicio que otros estudios plantean un nuevo modelo de percepciones expectativas con nuevas dimensiones, denominadas como aún más concretas, respecto de las utilizadas en los trabajos tradicioneles (Forero \& Gómez, 2017; Zapata, 2014).

Tabla 4 Resumen de artículos revisados

\begin{tabular}{|c|c|c|c|c|}
\hline Autor & Año & Muestra & País & Análisis estadístico \\
\hline Abuosi \& Atinga & 2013 & $\begin{array}{l}\mathrm{n}=250,50 \text { pacientes dados de alta en } \\
5 \text { hospitales públicos }\end{array}$ & Ghana & AFE \\
\hline $\begin{array}{l}\text { Al-Borie \& Sheikh } \\
\text { Damanhouri }\end{array}$ & 2013 & $\begin{array}{l}\mathrm{n}=1000, \text { pacientes en } 10 \text { hospitales } \\
\text { públicos y privados }\end{array}$ & $\begin{array}{l}\text { Arabia } \\
\text { Saudita }\end{array}$ & $\begin{array}{l}\text { Prueba t para comparación } \\
\text { de medias }\end{array}$ \\
\hline Almeida et al. & 2013 & $\begin{array}{l}\mathrm{n}=95 \text {, pacientes en área especializada } \\
\text { en un Hospital Universitario }\end{array}$ & Brasil & $\begin{array}{l}\text { Prueba chi cuadrado; } \\
\text { coeficiente Rho Spearman }\end{array}$ \\
\hline Garrard \& Narayan & 2013 & $\mathrm{n}=364$ pacientes en área de obstetricia & EEUU & Prueba de rangos Wilcoxon \\
\hline Miranda et al. & 2013 & $\begin{array}{l}\mathrm{n}=644 \text { pacientes víctimas de } \\
\text { accidentes de tránsito }\end{array}$ & Perú & Kruskal Wallis y chi cuadrado \\
\hline Purcărea et al. & 2013 & $\begin{array}{l}\mathrm{n}=183 \text { pacientes mujeres área } \\
\text { de ginecología }\end{array}$ & Rumania & AFE \\
\hline Rocha et al. & 2013 & $\begin{array}{l}\mathrm{n}=116 \text { pacientes en área de } \\
\text { cirugía plástica }\end{array}$ & Brasil & Correlación de Pearson \\
\hline $\begin{array}{l}\text { Yousapronpaiboon } \\
\text { \&Johnson }\end{array}$ & 2013 & $\begin{array}{l}\mathrm{n}=400 \text { pacientes hospitales } \\
\text { públicos y privados }\end{array}$ & Tailandia & AFC-SEM \\
\hline Aghamolaei et al. & 2014 & $\begin{array}{l}\mathrm{n}=89 \text { pacientes de Hospital } \\
\text { de Bansar }\end{array}$ & Irán & $\begin{array}{l}\text { Prueba de rangos Wilcoxon } \\
\text { y prueba de Kruskal-Walls }\end{array}$ \\
\hline Khamis \& Njau & 2014 & $\mathrm{n}=422$ pacientes en un hospital público & Tanzania & $\begin{array}{l}\text { Prueba t para comparación } \\
\text { de medias }\end{array}$ \\
\hline Zapata & 2014 & $\begin{array}{l}n=1250 \text { pacientes en servicios } \\
\text { de salud de Manizales }\end{array}$ & Colombia & AFC-SEM \\
\hline Al-Momani & 2015 & $\mathrm{n}=1500$ pacientes hospitalizados & $\begin{array}{l}\text { Arabia } \\
\text { Saudita }\end{array}$ & $\begin{array}{l}\text { Prueba t para comparación } \\
\text { de medias y ANOVA }\end{array}$ \\
\hline
\end{tabular}




\begin{tabular}{|c|c|c|c|c|}
\hline Koley et al. & 2015 & $\begin{array}{l}\mathrm{n}=644 \text { pacientes de hospitales } \\
\text { públicos homeopáticos }\end{array}$ & India & $\begin{array}{l}\text { Prueba t para comparación } \\
\text { de medias }\end{array}$ \\
\hline Li etal. & 2015 & $\begin{array}{l}\mathrm{n}=1963 \text { pacientes de hospitales } \\
\text { en cinco ciudades }\end{array}$ & China & $\begin{array}{l}\text { Pruebas de fiabilidad y } \\
\text { validez, correlación producto } \\
\text { momento Pearson, Prueba t } \\
\text { independiente de medias, } \\
\text { Regresión simultánea }\end{array}$ \\
\hline $\begin{array}{l}\text { Jandavath \& } \\
\text { Byram }\end{array}$ & 2016 & $\begin{array}{l}\mathrm{n}=493 \text { pacientes admitidos en el } \\
\text { terciario corporativo de hospitales } \\
\text { de atención en Chennai }\end{array}$ & India & AFC-SEM \\
\hline Kalaja et al. & 2016 & $\begin{array}{l}\mathrm{n}=200 \text { pacientes hospitalizados } \\
\text { en hospital público de Durres }\end{array}$ & Albania & Estadística descriptiva \\
\hline Nadi et al. & 2016 & $\begin{array}{l}\mathrm{n}=622 \text { pacientes en cinco hospitales } \\
\text { seleccionados }\end{array}$ & Irán & $\begin{array}{l}\text { Prueba Wilcoxon, } \\
\text { Prueba t y Friedman }\end{array}$ \\
\hline Cengiz \& Fidan & 2017 & $\mathrm{n}=308$ pacientes en hospital privado & Turquía & $\mathrm{AFE}-\mathrm{AFC}$ \\
\hline Fan et al. & 2017 & $\begin{array}{l}\mathrm{n}=1303 \text { pacientes en } 27 \text { hospitales } \\
\text { de } 15 \text { provincias }\end{array}$ & China & $\begin{array}{l}\text { T- pareado, regresión } \\
\text { binaria logística }\end{array}$ \\
\hline Forero \& Cómez & 2017 & n = 2954 pacientes en Bogotá y Cali & Colombia & $\mathrm{AFC}-\mathrm{SEM}$ \\
\hline Owusu Kwateng & 2017 & $\begin{array}{l}\mathrm{n}=400 \text { pacientes en hospitales } \\
\text { públicos y privados excluyendo } \\
\text { áreas de especializaciones }\end{array}$ & Turquía & $\begin{array}{l}\text { Estadística descriptiva, } \\
\text { prueba t }\end{array}$ \\
\hline Adepoju et al. & 2018 & $\mathrm{n}=239$ pacientes en APS & Nigeria & $\begin{array}{l}\text { Estadística descriptiva, } \\
\text { regresión múltiple }\end{array}$ \\
\hline Zun etal. & 2018 & $\mathrm{n}=430$ pacientes en 9 clínicas & Malasia & Prueba t, regresión logística \\
\hline Costa et al. & 2018 & $\begin{array}{l}\mathrm{n}=172 \text { pacientes internados más } \\
\text { de } 24 \text { horas }\end{array}$ & Brasil & $\begin{array}{l}\text { Prueba Wilcoxon, } \\
\text { coeficiente Kappa }\end{array}$ \\
\hline Musa etal. & 2018 & $\mathrm{n}=100$ pacientes en área de cirugía & Croacia & $\mathrm{AFE}$ \\
\hline
\end{tabular}

Por otro lado, otras técnicas estadísticas fueron utilizadas recurrentemente con objetivos en común. En general, los estudios que pretenden establecer diferencias entre las percepciones y expectativas obtuvieron resultados similares donde las percepciones de los pacientes se reportaron significativamente más bajas que sus expectativas (Fan et al., 2017), análisis llevados a cabo mayoritariamente mediante pruebas de rangos Wilcoxon (Garrard \& Narayan, 2013; Nadi et al., 2016 Aghamolaei et al., 2014) y prueba t para comparación de medias (Khamis \& Njau, 2014; Al-Momani, 2015; Koley et al., 2015), con una excepción en Costa et al. (2018) quienes encontraron que en algunas casos las percepciones superaron las expectativas, verificando así la sensibilidad de las escala.

Otros análisis frecuentes fueron la comparación de la satisfacción entre hospitales públicos y privados, donde la mayoría de estudios encontró una brecha mayor en la calidad de hospitales estatales (Al-Borie \& Sheikh Damanhouri, 2013; Miranda et al, 2013; Owusu Kwateng, 2017).

Además, se realizan comparaciones entre datos sociodemográficos, con coincidencias en la no asociación entre satisfacción y factores como género y educación (Aghamolaei et al., 2014; Li et al., 2015), mientras la edad podría resultar un predictor importante en la satisfacción (Almeida et al., 2013; Li et al., 2015).

\section{Síntesis conceptual y hallazgos documentales}

Del análisis realizado se logró sintetizar el contenido en las secciones calidad de servicio, elementos que conforman el constructo satisfacción como medida de calidad de servicio y una síntesis conceptual que da forma a la una base teórica de los modelos de percepción de calidad de servicio.

Seguidamente seprofundizóeneldenominado Modelo Servqual acompañado de una conceptualización del modelo asociado además a los Gaps en la calidad del servicio, mismos que, de una o de otra forma, se estructuran sobre la base de factores que componen y constituyen el denominado Método Servqual cuyos elementos de medición sistematizan el modelo Servqual.

Finalmente se sintetiza algunas ventajas del Método SERVQUAL y, por supuesto también, algunas desventajas del Método que diversos analistas han presentado en torno al SERVQUAL. 


\section{Calidad de Servicio}

Los primeros esfuerzos por definir la calidad del servicio se inclinan a hacerlo de acuerdo con los criterios del prestatario, con la consciente adaptación a las especificaciones definidas por el productor. Prueba de esto es que en 1972, Levitt propone aplicar a la producción de los servicios definiciones y técnicas de control de calidad similares a las de la manufactura (Losada et al., 2007). A este enfoque de la calidad se le conoce como calidad objetiva o experimentada (Figura 2), que se refiere a la superioridad medible y verificable del servicio frente a un ideal estándar según señala Zeithaml, (1988) (Dolors, 2014).

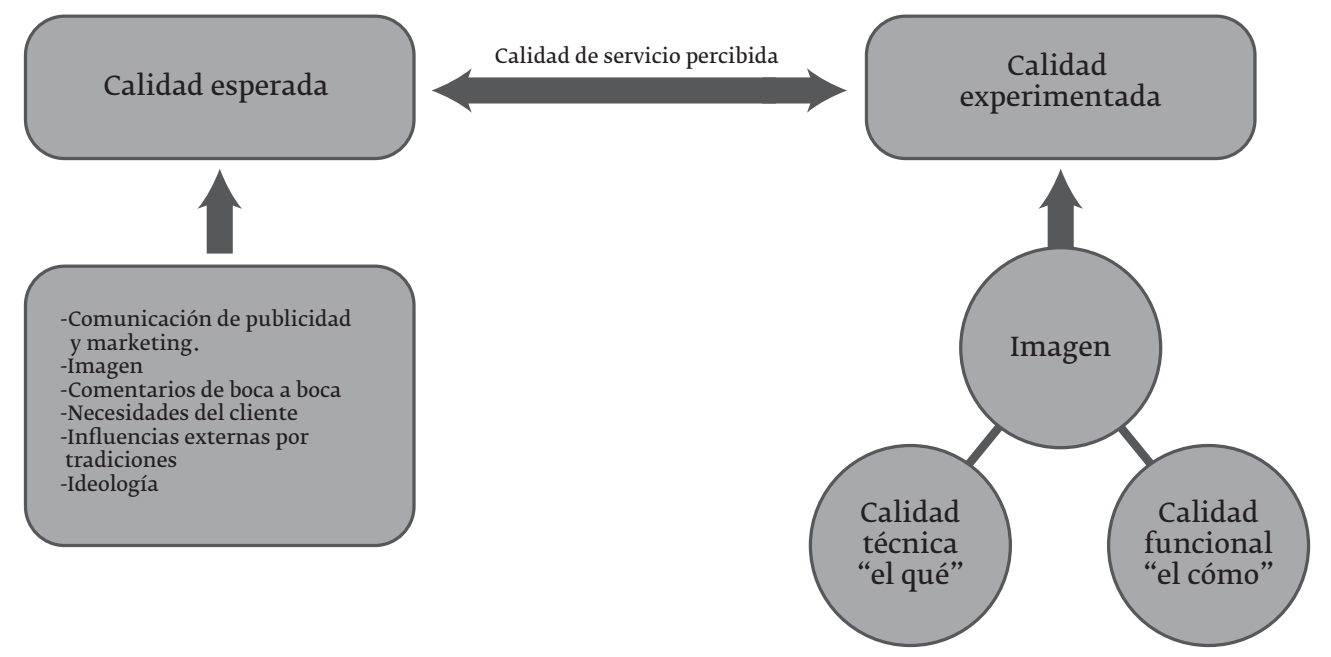

Figura 1: Modelo de calidad percibida de Gronroos. Adaptado de Gronroos, C. (1984). A service quality model and its marketing implications. European Journal of marketing, 18(4), 36-44.

Un cambio en la visión objetiva de la calidad del servicio se gesta a partir de Gronroos (1982; 1984), quien manifiesta que dada su inmaterialidad, los servicios requieren una activa interacción entre el comprador y el proveedor. Esta relación origina el concepto de calidad del servicio percibida, que implica el juicio subjetivo del cliente sobre el servicio que recibe. Introducir la percepción conlleva formular el concepto de calidad del servicio desde la óptica del cliente señalado por Gil en 1995 (Dolors, 2014).

Zaithaml 1988 y Bitrner 1990 señalan que la calidad de servicio percibida por el cliente puede ser conceptualizada como una valoración global, altamente subjetiva, similar a una actitud, relacionada pero no equivalente a la satisfacción, que implica un proceso de valoración continuada en el tiempo. Además define la calidad como la superioridad o excelencia, donde la calidad percibida se puede entender como el juicio de un consumidor acerca de la excelencia total o superioridad de un producto o servicio. Este concepto es diferente de la calidad objetiva, ya que vincula la perspectiva del consumidor/comprador; haciendo referencia a un nivel superior de abstracción, más que a un atributo especifico del producto o servicio; es una evaluación global similar a una actitud relacionada pero no equivalente a la satisfacción, y corresponde a un juicio hecho usualmente dentro de un conjunto evocado por el consumidor (Losada et al., 2007).

\section{El constructo satisfacción como medida de calidad de servicio}

Las relaciones entre la calidad de servicio $y$ la satisfacción del consumidor o usuaria han sido extensamente discutidas, en especial por Parasuraman et al. (1988) y Cronin y Baker (1994). Parte de la literatura sugiere que la calidad de servicio y la satisfacción del consumidor son conceptos interdependientes, en tanto y en cuanto éstos son constructos que comparten una relación conceptual y empírica cercana (Cronin \& Taylor, 1992).

La satisfacción usuaria fue en primera instancia vista simplemente como una respuesta del cliente al cumplimiento con su demanda (Losada et al., 2007). Sin embargo, Oliver (1993) argumentó que la satisfacción no solo corresponde a un sentimiento de saciedad, sino que más bien puede ser descrito como un proceso complejo. En consecuencia, sobre la base de este enfoque, se plantea que la satisfacción puede estar determinada por dos factores: (1) una respuesta emocional afectiva positiva o negativa y (2) una aprobación o rechazo cognitivo. De hecho, ya anteriormente Oliver (1981) había definido la satisfacción como un resumen del estado psicológico cuando las expectativas no cubiertas del consumidor se enlazan con un sentimiento a priori sobre la experiencia del consumo; en concreto, el autor supone que la satisfacción es, en definitiva una actitud.

Por el contrario, otros estudios manifiestan que la calidad de servicio ha mostrado ser consistente en la literatura como un constructo único derivado de la satisfacción del consumidor (Cronin y Baker, 1994). Complementariamente, Parasuraman et al. (1985) la observan como un juicio global relacionado con la superioridad de un servicio en general, mientras la satisfacción refiere tan solo a una situación específica autopercibida. Para el soporte de esta aseveración otros estudios (Taylor, 1993) han aportado elementos claves para la distinción entre los dominios de calidad de servicio y satisfacción usuaria:

-Las dimensiones bajo los criterios de calidad son específicos, mientras la satisfacción puede resultar de una infinidad de dimensiones (sean o no relacionadas a la calidad).

- Las expectativas de calidad están basadas en ideales de excelencia del servicio, mientras un gran número 
de aspectos no relacionados a la calidad pueden formar los criterios de satisfacción (por ejemplo necesidades, equidad, percepciones, etc).

- Las percepciones de calidad no requieren un experiencia pasada con el proveedor del servicio, mientras los criterios de satisfacción sí.

- La calidad tiene más antecedentes conceptuales que el constructo de satisfacción.

Sin embargo, un número importante de evidencia respecto al tema de servicios apoya la postura de que la calidad y la satisfacción del cliente se conceptualizan de mejor manera como un único constructo, pero no deberían ser tratados como equivalentes en modelos de toma de decisión de consumidores (Cronin y Baker, 1994).

En esta idea, se puede señalar que los dos constructos están relacionados, y consecuentemente la satisfacción a través del tiempo resulta ser una variable latente de la percepción efectivamente observable de la calidad de un servicio (Parasuraman et al., 1985).

\section{Base teórica de modelos de percepción de calidad de servicio}

Los modelos teóricos que involucran conceptos de expectativa y percepción surgen de las investigaciones seminales de Gronroos (1984) y Parasuraman, Berry y Zeithmal (1985).

Por una parte, las teorías sobre calidad de servicio nacen por las características particulares del mismo. Gronroos (1988) explicaba que un servicio posee atributos únicos que lo distinguen de cualquier otro bien, estos serían: (1) su intangibilidad, (2) su forma simultánea de producción y consumo (3) es una actividad en lugar de objeto; y (4) los consumidores son partícipes del proceso de producción. Por otra parte, Grönroos (1984) señaló que las organizaciones deben tener la capacidad de influir en las percepciones de los consumidores y deben gestionar la calidad del servicio buscando reducir la brecha (Figura 3) entre las expectativas (ideales, y las percepciones (auto obtenidas) de los consumidores.

En este mismo enfoque Parasuraman, Berry y Zeithmal (1985) elaboraron otro modelo teórico de calidad de servicio. Este modelo presenta diez dimensiones y es conocido también como el modelo de gaps o modelo de brechas, pues el modelo describe la calidad como la diferencia entre la expectativa y la percepción real del servicio dado.

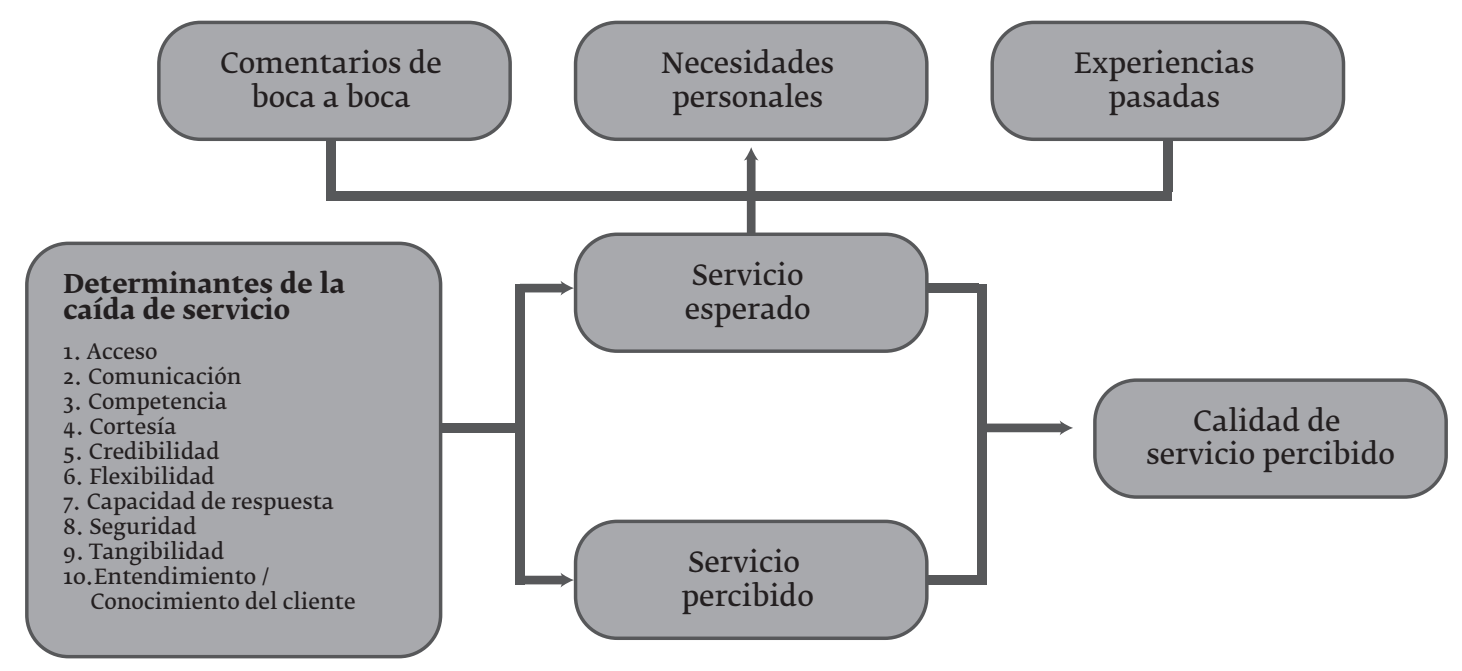

Figura 3: Modelo de brechas o gaps. Adaptado de Parasuraman, Zeithmail y Berry (1985).

A conceptual model of service quality and its implications for future research. The Journal of Marketing

Posteriormente estas diez dimensiones fueron reducidas a cinco. Las dimensiones simplificadas del modelo de Parasuraman et al., (1985) que valoran la calidad de los servicios, los factores descritos se sintetizaron en factores que se agruparon en cinco (5) dimensiones claramente diferenciadas que contienen ítems convergentes (Parasuraman, Zeithaml, Berry, 1988). Estas dimensiones dan origen a un método denominado Modelo SERVQUAL cuyos descriptores son los siguientes:

- Confianza: Capacidad para desempeñar el servicio que se promete, de manera segura y precisa. -Responsabilidad: Disponibilidad para ayudar a los clientes y para promover el servicio con prontitud. - Seguridad: Conocimiento y cortesía de los empleados y su habilidad para inspirar buena fe y confianza. - Empatía: Brindar a los clientas una atención individualizada y cuidadosa.
-Tangibles: Apariencia de las instalaciones físicas, el equipo, el personal y los materiales escritos.

Posterior a este modelo teórico surgen una variedad de adaptaciones propuestas por autores en diferentes contextos (Valencia, Piedrahita, Zapata, Benjumea \& Palacios, 2018; Teshnizi, Aghamolae, Kahnouji, Teshnizi y Ghani, 2018; Numpaque \& Rocha, 2016). De hecho, las adaptaciones al modelo SERVQUAL para medir la satisfacción de usuaria, en el caso particular de los servicios de salud, tienen más de 20 años de registro, que desde una perspectiva más bien empírica, se puede observar que en los últimos cinco años, diversos trabajos y en diferentes países, se busca confirmar si este modelo es una medida de calidad válida en el contexto de sus instituciones de salud. La literatura a su vez permite corroborar que las investigaciones que involucran los modelos de calidad percibida es abundante, lo que incluso ha 
dado paso a estudios que llevan a cabo meta análisis y otros tipos de análisis bibliométricos (Valencia, Piedrahita, Zapata, Benjumea \& Palacios, 2018; Teshnizi, Aghamolae, Kahnouji, Teshnizi y Ghani, 2018; Numpaque \& Rocha, 2016).

Con dichos antecedentes, el presente estudio estableció como objetivo sintetizar la evidencia científica relacionada con las adaptaciones y usos del modelo de Servqual además de esquematizar y clasificar algunas de las más frecuentes metodologías empleadas en las investigaciones de medición de la calidad de servicio.

\section{Modelo SERVQUAL}

En cuanto a la medida cuantitativa de la calidad del servicio (Figura 4), uno de los trabajos pioneros se recoge bajo la denominación de SERVQUAL (Díaz, 2005).

Este Modelo, es un procedimiento de evaluación que se realiza mediante la aplicación del método de encuesta y sobre la base de un cuestionario que busca medir un sistema de dimensiones y de ítems que representan los componentes de la calidad de servicio (Chen, Spohrer, \& Lelescu, 2008).

\section{SERVQUAL}

Parasuraman.Zeithaml y Berry $(1985$ - 1988)

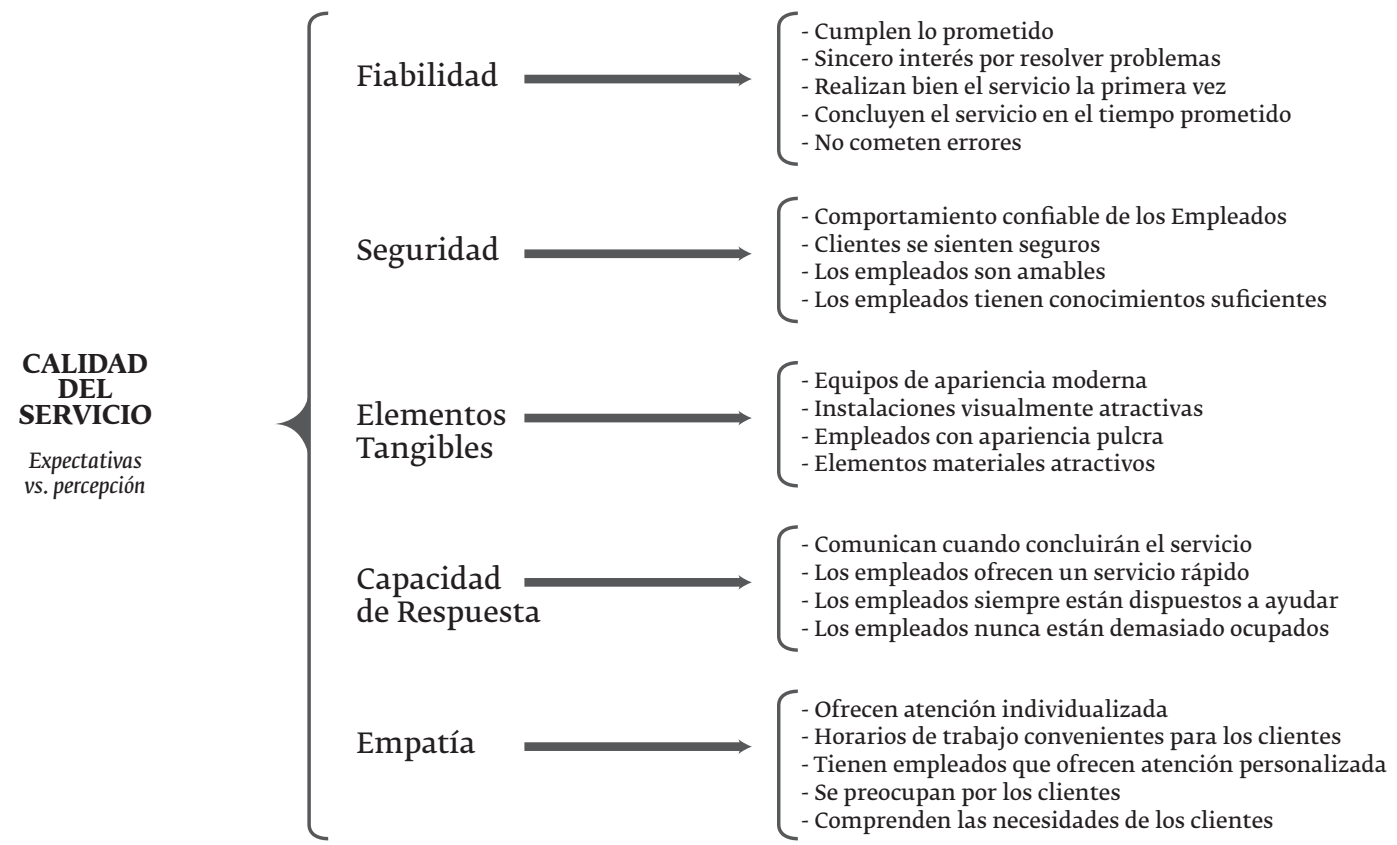

Figura 4: Modelo Servqual (Parasuraman, Zeithaml Y Berry) (1985-1988)

El cuestionario recoge las expectativas y percepciones (Edvardsson y Tronvoll, 20012; 2013) y en consecuencia determina la desviación que existe entre éstos agregados a partir de los registros en escala de Likert (1932) respecto de un determinado producto o servicio relevante para los diversos grupos de interés que la organización ofertante atiende (Frow y Payne, 2011). Además, SERVQUAL, de acuerdo con la diversidad de fuentes consultadas (Jadika, Khoo y Na, 2011; Pautasso, 2013), confirma la existencia de un número de 22 preguntas que ponen en evidencia cinco dimensiones o factores latentes (Parasuraman, Zeithaml Y Berry) (1985-1988) relativos a un sistema de criterios de evaluación de la calidad por parte de los clientes a un producto o servicio (Meyer y Schwager, 2007). En consecuencia, el cuestionario SERVQUAL diseñado por Parasuraman (Parasuraman, Zeithaml, Berry, 1988), por su validez y confiabilidad (Zhuang, 2010), es la herramienta multidimensional más aceptada y utilizada para medir la calidad de atención en empresas de servicios (Wolniak, SkotnickaZasadzien, 2011) e incorpora en su diseño (Figura 2) un conjunto de requisitos de calidad (Cabello y Chirinos, 2012).

\section{Conceptualización de los Gaps del Modelo SERVQUAL}

En el modelo SERVQUAL se distinguen dos partes claramente diferenciadas, cliente - compañía (Figura 5) pero relacionadas entre sí: 


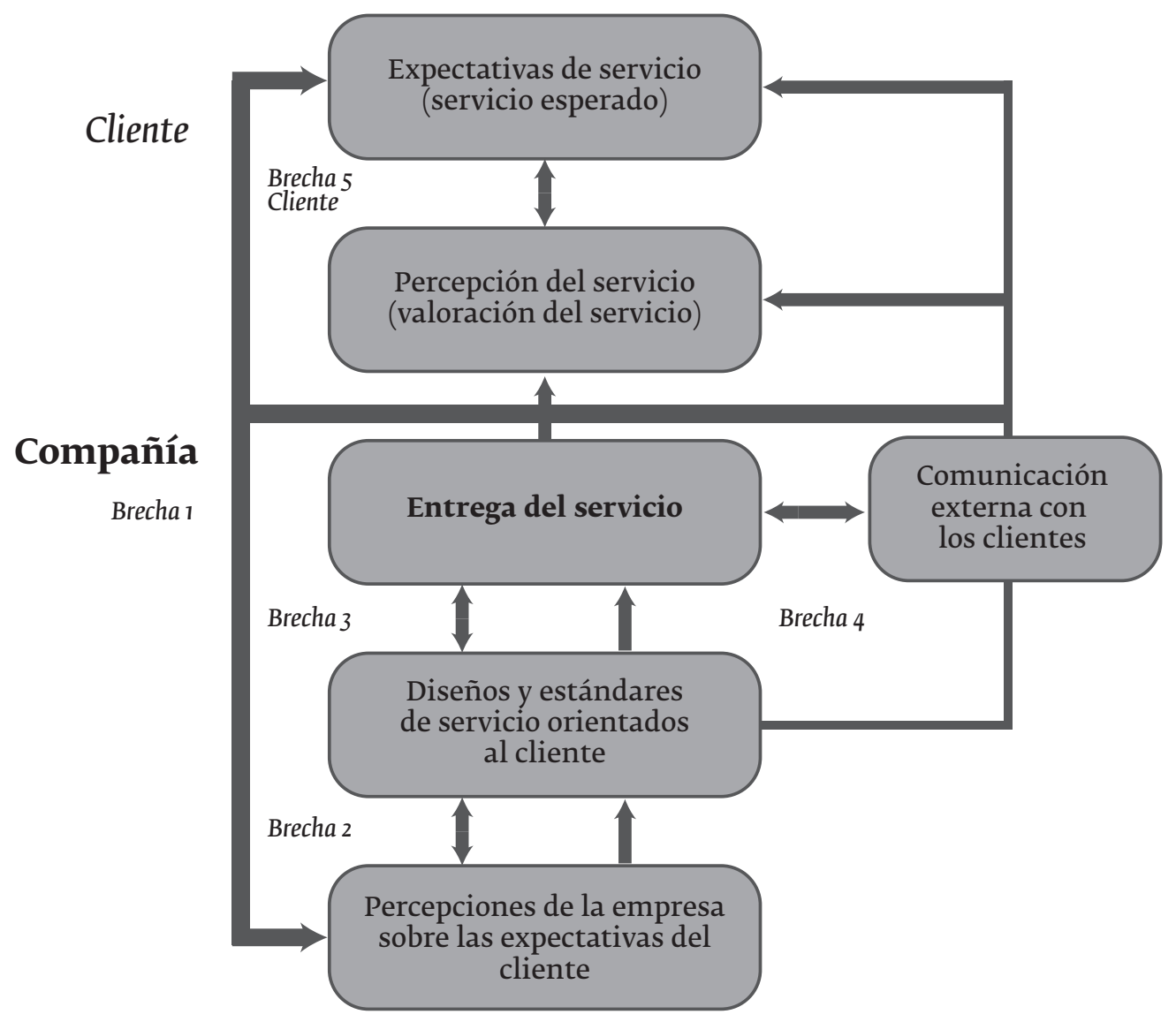

Figura 5: Modelo de las brechas sobre la calidad en el servicio (Zeithaml, V, Mary Jo Bitner, 2002)

Como puede apreciarse en la Figura 5, el modelo introduce y analiza una serie de discrepancias, diferencias o gaps que pueden ser percibidas por los clientes (gap 5), o bien producirse internamente en las organizaciones proveedoras de los servicios (gaps del 1 al 4) (Parasuraman, Zeithaml, y Berry, 1985).

La primera hace referencia a la manera en que los clientes se forman una opinión sobre la calidad de los servicios recibidos (parte superior de la figura 5). La segunda refleja las deficiencias que pueden producirse dentro de las organizaciones, lo que provoca una falta de calidad en el suministro a los clientes (parte inferior de la figura 5).

-Gap 1: indica la discrepancia entre las expectativas de los clientes sobre un servicio concreto y las percepciones o creencias que se forman los directivos sobre lo que espera el consumidor de ese servicio.

-Gap 2: mide la diferencia entre las percepciones de los directivos y las especificaciones o normas de calidad. -Gap 3: calcula la diferencia entre las especificaciones o normas de calidad del servicio y la prestación del mismo. -Gap 4: mide la discrepancia entre la prestación del servicio y la comunicación externa.

- Gap 5: mide la diferencia entre el servicio esperado y el servicio percibido, determinando a través de dicha magnitud el nivel de calidad alcanzado. La forma de reducir esta diferencia es controlando $y$ disminuyendo todas las demás.

GAP $5=f(G A P$ 1, GAP 2, GAP 3, GAP 4)

\section{Elementos de medición de los componentes del Método SERVQUAL}

La primera hace referencia a la manera en que los clientes se forman una opinión sobre la calidad de los servicios recibidos (parte superior de la figura 5). La segunda refleja las deficiencias que pueden producirse dentro de las organizaciones, lo que provoca una falta de calidad en el suministro a los clientes (parte inferior de la figura 5).

El Método SERVQUAL requiere la opinión de los clientes respecto de los ítems del cuestionario. Es por esta razón que se puede afirmar que el modelo corresponde a un cuestionario estructurado, con preguntas cerradas que aborda 5 dimensiones $o$ variables latentes que agrupan 22 ítems de calidad de servicio. Este cuestionario está compuesto por tres partes, la cuales serán detalladas a continuación:

-La primera parte, tiene como objetivo medir las expectativas del consumidor frente a un servicio determinado, mediante la realización de afirmaciones respeto a como debiera ser el servicio prestado por una organización ideal del mismo tipo, o sea lo esperado

- La segunda parte, mide la percepción del cliente respecto a la calidad del servicio brindado, tomando como base, el desempeño efectivo de esa organización, lo que debe quedar claro es que persona 
evaluada debe conocer la organización o haber tenido alguna experiencia de ésta.

- La tercera sección, pretende cuantificar las evaluaciones de los clientes respecto la importancia relativa de las 5 dimensiones.

Es necesario mencionar, que este cuestionario se puede adaptar a las necesidades de medición de un estudio específico así como a las características de la organización dónde se aplique. La medición del cuestionario SERVQUAL se puede realizar mediante la Escala de Likert, la cual es denominada así en honor de Rensis Likert, quien diseñó este método en 1932 (Likert, 1932).

El instrumento presenta al cliente o usuario una serie de 22 proposiciones, ante las cuales el individuo debe mostrar un cierto grado de "acuerdo" registrando su opinión en la escala Likert propuesta, normalmente de 5 puntos creciente. La escala es de tipo ordinal, de medición compuesta, en tanto mide tanto la percepción de satisfacción usuaria y la expectativa de satisfacción usuaria mediante una serie de parámetros (Parasuraman, Zeithaml y Berry, 1991). El grado de satisfacción se puede medir, por ejemplo, aplicando el siguiente criterio:

- Bajo o menor a $60 \%$

- Mediano o aceptable entre $60 \%$ a $70 \%$

- Superior o bueno entre $70 \%$ a $85 \%$

- Alto entre $85 \%$ a $100 \%$ $\rightarrow 4,20$

4,20 a 4,90

4,90 a 5,59

5,59 a 7,00

\section{Ventajas del Método SERVQUAL}

Este método permite realizar una comparación de las expectativas de los clientes y las percepciones de los clientes de cualquier organización en un tiempo determinado y con ello lograr establecer similitudes y diferencias y así poder realizar diagnósticos de la calidad de servicio. También se pueden efectuar comparaciones entre diferentes organizaciones que presten el mismo servicio en otras localidades. Es un método fácil de adaptar, el cual se puede modificar de acuerdo a las características de la organización que se desea evaluar. Por último permite examinar la diversidad de opiniones de los clientes, percepciones y expectativas relacionadas con la calidad de servicio.

\section{Desventajas del Método SERVQUAL}

Este método se realiza a través de cuestionarios por lo tanto la información puede estar sesgada, debido a que las personas pueden no entender la dinámica del cuestionario, y por ello no saber con certeza si el significado de las preguntas es totalmente claro. Además, este cuestionario cuenta con 5 dimensiones y 22 atributos en total, lo que al evaluar las percepciones y las expectativas, puede ser tedioso al usuario de responder la totalidad de 44 preguntas.

\section{Estudios en Calidad de Servicio}

Con el objetivo de mostrar cómo y de qué manera el constructo de la calidad de servicio ha sido aplicada, se ha logrado identificar un número importante de evidencia que se detalla sintéticamente en la tabla 5.

\begin{tabular}{|c|c|c|c|c|}
\hline Autor & Modelo & Muestra evaluada & Método usado & Escala de medida \\
\hline Gronroos (1984) & $\begin{array}{l}\text { Calidad técnica } \\
\text { y funcional }\end{array}$ & $\begin{array}{l}219 \text { encuestados de organizaciones } \\
\text { de bancos, seguros, restaurantes, } \\
\text { aeropuertos, renta de autos, agencias } \\
\text { de viajes y otras instituciones públicas }\end{array}$ & $\begin{array}{l}\text { Análisis estadístico } \\
\text { básico }\end{array}$ & Calidad técnica y calidad funcional \\
\hline $\begin{array}{l}\text { Parasuraman } \\
\text { et al. (1985) }\end{array}$ & $\begin{array}{l}\text { Modelo de brechas } \\
\text { ogaps }\end{array}$ & $\begin{array}{l}\text { Entre } 298 \text { a } 487 \text { encuestados de compañías } \\
\text { de telefonía, seguros, bancos, } \\
\text { mantenimiento. }\end{array}$ & $\begin{array}{l}\text { Factor de eje principal seguido } \\
\text { de rotación oblicua }\end{array}$ & $\begin{array}{l}\text { Diez dimensiones (confiabilidad, } \\
\text { seguridad, receptividad, acceso, } \\
\text { comunicación, tangibles, cortesía, } \\
\text { credibilidad, competencia y } \\
\text { comprensión / conocimiento }\end{array}$ \\
\hline $\begin{array}{l}\text { Haywood \& } \\
\text { Farmer (1988) }\end{array}$ & $\begin{array}{l}\text { Modelo de atributos } \\
\text { de calidad de servicio }\end{array}$ & & No reportado & $\begin{array}{l}\text { Instalaciones y procesos físicos, } \\
\text { comportamiento y cordialidad } \\
\text { de las personas, y juicio profesional }\end{array}$ \\
\hline $\begin{array}{l}\text { Brogowicz et al. } \\
\text { (1990) }\end{array}$ & $\begin{array}{l}\text { Modelo sintetizado } \\
\text { de calidad de servicio }\end{array}$ & & No reportado & $\begin{array}{l}\text { Calidad técnica y funcional que } \\
\text { define tareas de planificación, } \\
\text { implementación y control }\end{array}$ \\
\hline Cronin \& Taylor & $\begin{array}{l}\text { Modelo de } \\
\text { rendimiento }\end{array}$ & $\begin{array}{l}\text { 66o encuestados de compañías } \\
\text { de bancos, lavadoras y comida rápida }\end{array}$ & $\begin{array}{l}\text { Factor de eje principal } \\
\text { seguido de rotación oblicua } \\
\text { en LISREL }\end{array}$ & $\begin{array}{l}\text { SERVQUAL solo con ítems de } \\
\text { percepción(SERVPERF) }\end{array}$ \\
\hline Mattsson (1992) & $\begin{array}{l}\text { Modelo ideal } \\
\text { de valor }\end{array}$ & 40 huéspedes de hotel & $\begin{array}{l}\text { Correlación de momentos } \\
\text { de Pearson, intravesa por } \\
\text { pares y prueba de la mediana } \\
\text { entre muestras, y prueba } \\
\text { de chi-cuadrado }\end{array}$ & $\begin{array}{l}18 \text { ítems de valor y } 9 \text { ítems de } \\
\text { satisfacción del cliente }\end{array}$ \\
\hline Teas (1993) & $\begin{array}{l}\text { Modelo de calidad } \\
\text { normaday } \\
\text { rendimiento } \\
\text { evaluado }\end{array}$ & 120 encuestados de tiendas retail & $\begin{array}{l}\text { Evaluación cualitativa, } \\
\text { correlación y prueba t }\end{array}$ & $\begin{array}{l}\text { Conjunto limitado de elementos } \\
\text { SERVQUAL ( } 2 \text { ítems para cada una } \\
\text { de } 5 \text { dimensiones) }\end{array}$ \\
\hline $\begin{array}{l}\text { Dabholkar etal. } \\
\text { (1996) }\end{array}$ & $\begin{array}{l}\text { Modelo de atributos } \\
\text { y afecto total }\end{array}$ & $\begin{array}{l}505 \text { estudiantes de locales de } \\
\text { comida rápida }\end{array}$ & $\begin{array}{l}\text { Análisis factorial confirmatorio } \\
\text { y modelado de ecuaciones } \\
\text { estructurales usando LISREL VII }\end{array}$ & $\begin{array}{l}3 \text { elementos que miden la calidad } \\
\text { del servicio esperado, específicamente } \\
\text { de la situación de pedido }\end{array}$ \\
\hline
\end{tabular}




\begin{tabular}{|c|c|c|c|c|}
\hline $\begin{array}{l}\text { Spreng \& Mackoy } \\
\text { (1996) }\end{array}$ & $\begin{array}{l}\text { Modelo de calidad } \\
\text { percibida y satisfacción }\end{array}$ & 273 estudiantes & $\begin{array}{l}\text { Análisis factorial confirmatorio } \\
\text { y modelado de ecuaciones } \\
\text { estructurales usando LISREL VII }\end{array}$ & $\begin{array}{l}\text { Deseos, rendimiento percibido, } \\
\text { expectativas y congruencia deseada } \\
\text { (cada uno con 1o atributos) }\end{array}$ \\
\hline $\begin{array}{l}\text { Phillip \& Hazlett } \\
\text { (1997) }\end{array}$ & $\begin{array}{l}\text { Modelo de atributos } \\
\text { global }\end{array}$ & & No reportado & $\begin{array}{l}\text { Atributos fundamentales, } \\
\text { atributos principales y } \\
\text { atributos periféricos }\end{array}$ \\
\hline $\begin{array}{l}\text { Sweeney et al. } \\
\text { (1997) }\end{array}$ & $\begin{array}{l}\text { Modelo de calidad } \\
\text { de servicio y valor } \\
\text { percibido para retail }\end{array}$ & $\begin{array}{l}1016 \text { clientes de tiendas de } \\
\text { electrodomésticos }\end{array}$ & $\begin{array}{l}\text { Análisis factorial } \\
\text { confirmatorio en LISREL }\end{array}$ & $\begin{array}{l}\text { Calidad funcional a través de } 5 \\
\text { elementos SERVQUAL y calidad } \\
\text { técnica a través de } 1 \text { ítem }\end{array}$ \\
\hline Oh(1999) & $\begin{array}{l}\text { Modelo de calidad } \\
\text { percibida, valor de } \\
\text { clientey satisfacción } \\
\text { de cliente }\end{array}$ & 545 huéspedes dos hoteles de lujo & Análisis Path en LISREL VIII & $\begin{array}{l}\text { Ítem único para el precio percibido } \\
\text { y } 8 \text { ítems para las percepciones de } \\
\text { la configuración del hotel }\end{array}$ \\
\hline $\begin{array}{l}\text { Dabholkar et al. } \\
(2000)\end{array}$ & $\begin{array}{l}\text { Modelo de mediador } \\
\text { antecedente }\end{array}$ & 397 estudiantes & $\begin{array}{l}\text { Regresiones en modelo de } \\
\text { ecuaciones estructurales } \\
\text { en LISREL }\end{array}$ & $\begin{array}{l}\text { SERVQUAL a través de la medición } \\
\text { de confiabilidad, atención personal, } \\
\text { comodidades y características }\end{array}$ \\
\hline $\begin{array}{l}\text { Frost \& Kumar } \\
(2000)\end{array}$ & $\begin{array}{l}\text { Modelo de calidad } \\
\text { de servicio interno }\end{array}$ & 742 clientes de aerolíneas en Singapur & $\begin{array}{l}\text { Análisis de componentes } \\
\text { principales, coeficientes } \\
\text { de fiabilidad }\end{array}$ & SERVQUAL \\
\hline Dagger et al. (2007) & $\begin{array}{l}\text { Modelo de calidad } \\
\text { de servicio de salud }\end{array}$ & $\begin{array}{l}28 \text { participantes de grupos } \\
\text { focales en clínicas }\end{array}$ & Análisis cualitativo & $\begin{array}{l}\text { Calidad interpersonal, calidad } \\
\text { técnica, calidad ambiental y } \\
\text { calidad administrativa }\end{array}$ \\
\hline $\begin{array}{l}\text { Rakhmawati } \\
\text { etal. (2013) }\end{array}$ & $\begin{array}{l}\text { Modelo de calidad } \\
\text { de servicio para un } \\
\text { centro de salud pública }\end{array}$ & 8oo pacientes & Análisis factorial & $\begin{array}{l}\text { Calidad de la prestación de servicios } \\
\text { de salud, calidad del personal de } \\
\text { atención médica, adecuación de los } \\
\text { recursos de atención médica y calidad } \\
\text { del proceso de administración }\end{array}$ \\
\hline Lee (2016) & HEALTHQUAL & 757 pacientes & Análisis factorial & $\begin{array}{l}\text { Empatía, tangibles, seguridad, } \\
\text { eficiencia y grado de mejoras en el } \\
\text { servicio de atención }\end{array}$ \\
\hline
\end{tabular}

\section{Aplicaciones de la calidad de servicio en salud}

Las adaptaciones del modelo Servqual para medir la satisfacción de usuaria en los servicios de salud tienen más de 20 años de registro. Sin embargo, una revisión empírica permite corroborar que en los últimos cinco años, en diferentes países alrededor del mundo, se busca confirmar si este modelo es una medida de calidad válida en dicho contexto. Complementariamente y sobre la base de una definición ofrecida por Donabedian (1980), se establece que una atención médica de calidad es "un tipo de atención que maximiza el bienestar del paciente...” (Galván, Moctezuma, Dolci \& López, 2016). Por lo que, la concepción de que el paciente es el factor determinante en el nivel de calidad de servicio ofrecida, comienza a tomar forma en las instituciones del sector de la salud. De manera que se requiere de un cierto método de evaluación que permita medir objetivamente dicha calidad, como el que se presenta en la tabla 6.

Tabla 6 Métodos de evaluación de calidad de atención médica según el enfoque de Donabedian

\begin{tabular}{ll}
\hline Estructura & $\begin{array}{l}\text { Denota los atributos de las instalaciones en las que el servicio médico ocurre. Estos incluyen los atributos de los recursos materiales, } \\
\text { recursos humanos y la estructura de la organización. }\end{array}$ \\
\hline Proceso & $\begin{array}{l}\text { Denota las actividades durante la entrega y recepción del servicio médico. Incluye las actividades del paciente al buscar el servicio y las } \\
\text { actividades de los profesionales médicos en el diagnóstico, recomendaciones e implementación del tratamiento. }\end{array}$ \\
\hline Resultado & $\begin{array}{l}\text { Denota los efectos del cuidado médico sobre el estado de salud del paciente. Mejoras en el conocimiento del paciente sobre aspectos } \\
\text { sanitarios y su comportamiento. }\end{array}$ \\
\hline
\end{tabular}

Adaptado de Donabedian, A. (1997). The quality of care: how can it be assessed?

Archives of pathology \& laboratory medicine

Por último, la tabla 7 presenta en orden cronológico las investigaciones realizadas cuyo principal objetivo ha sido adaptar y validar el instrumento Servqual en hospitales e instituciones prestadoras de servicios de salud en diferentes ciudades o países.

Tabla 7 Esquematización de estudios referentes a calidad de servicio en atención médica

\begin{tabular}{|c|c|c|c|c|c|}
\hline Año & Artículo & Autores & Revista & Muestra & Ciudad o país \\
\hline 1990 & $\begin{array}{l}\text { Exploring perceptions of } \\
\text { hospital operations by a } \\
\text { modified SERVQUAL approach. }\end{array}$ & $\begin{array}{l}\text { Reidenbach, R. E., \& } \\
\text { Sandifer-Smallwood, B }\end{array}$ & Marketing Health Services & $\begin{array}{l}300 \text { pacientes de sala de } \\
\text { emergencia, externos } \\
\text { e internos }\end{array}$ & No reportado \\
\hline 1992 & $\begin{array}{l}\text { Adapting the SERVQUAL scale } \\
\text { to hospital services: an empirical } \\
\text { investigation. }\end{array}$ & $\begin{array}{l}\text { Babakus, E., \& } \\
\text { Mangold, W. C. }\end{array}$ & Health services research & 1999 pacientes dados de alta & No reportado \\
\hline
\end{tabular}




\begin{tabular}{|c|c|c|c|c|c|}
\hline 2008 & $\begin{array}{l}\text { Propuesta de una escala para medir } \\
\text { la calidad del servicio de los centros } \\
\text { de atención secundaria de salud. }\end{array}$ & $\begin{array}{l}\text { Torres Moraga, E., } \\
\text { \& Lastra Torres, J. }\end{array}$ & $\begin{array}{l}\text { Revista de Administração } \\
\text { Pública-RAP }\end{array}$ & 498 pacientes & $\begin{array}{l}\text { Santiago de Chile, } \\
\text { Chile }\end{array}$ \\
\hline 2010 & $\begin{array}{l}\text { Private healthcare quality: } \\
\text { applying a SERVQUAL model. }\end{array}$ & $\begin{array}{l}\text { Muhammad Butt, M., } \\
\text { \& Cyril de Run, E. }\end{array}$ & $\begin{array}{l}\text { International journal of } \\
\text { health care quality } \\
\text { assurance }\end{array}$ & 340 pacientes aleatorios & Malasia \\
\hline 2010 & $\begin{array}{l}\text { Validez y confiabilidad de do } \\
\text { SERVQHOS para enfermagem } \\
\text { em Boyacá, Colombia. }\end{array}$ & $\begin{array}{l}\text { Barragán Becerra, J. A. } \\
\text {, \& Manrique }\end{array}$ & Avances en Enfermería & 350 servicios quirúrgicos & Colombia \\
\hline 2012 & $\begin{array}{l}\text { Validación y aplicabilidad de } \\
\text { encuestas SERVQUAL } \\
\text { modificadas para medir la } \\
\text { satisfacción de usuarios externos } \\
\text { en servicios de salud. }\end{array}$ & $\begin{array}{l}\text { Cabello, E., \& } \\
\text { Chirinos, J. L. }\end{array}$ & Revista Médica Herediana & $\begin{array}{l}383 \text { usuarios de consulta } \\
\text { externa y } 384 \text { de emergencia }\end{array}$ & Perú \\
\hline 2014 & $\begin{array}{l}\text { Service quality assessment of a } \\
\text { referral hospital in Southern Iran } \\
\text { with SERVQUAL technique: } \\
\text { patients' perspective. }\end{array}$ & $\begin{array}{l}\text { Aghamolaei, T., } \\
\text { Eftekhaari, T. E., etal. }\end{array}$ & $\begin{array}{l}\text { BMC health services } \\
\text { research. }\end{array}$ & 96 participantes & Irán \\
\hline 2016 & $\begin{array}{l}\text { Service quality assessment in } \\
\text { health care sector: the case of } \\
\text { Durres public hospital }\end{array}$ & $\begin{array}{l}\text { Kalaja, R., Myshketa, } \\
\text { R., \& Scalera, F. }\end{array}$ & $\begin{array}{l}\text { Procedia-Social and } \\
\text { Behavioral Sciences }\end{array}$ & 200 pacientes hospitalizados & Durres, Albania \\
\hline 2018 & $\begin{array}{l}\text { Measuring patient's satisfaction } \\
\text { of healthcare services in the } \\
\text { UAE hospitals: Using SERVQUAL. }\end{array}$ & $\begin{array}{l}\text { Al-Neyadi, H. S., } \\
\text { Abdallah, S., } \\
\text { \& Malik, M. }\end{array}$ & $\begin{array}{l}\text { International Journal } \\
\text { of Healthcare Management, } \\
11(2), 96-105 \text {. }\end{array}$ & 217 pacientes & $\begin{array}{l}\text { Emiratos } \\
\text { Árabes Unidos }\end{array}$ \\
\hline
\end{tabular}

\section{Conclusiones}

Se logró indagar en la base teórica del concepto de calidad de servicio a través de la revisión bibliográfica exploratoria de publicaciones disponibles en bases de datos abiertas, lo que permitió observar su evolución abordando un periodo de más de 30 años desde su origen, con un cierto enfoque en los últimos cinco años, pasando de 10 publicaciones en 2013 a 15 publicaciones en 2018.

Desde la perspectiva empírica se pudo verificar cómo diversos modelos de análisis fueron sistemáticamente validados en diversas regiones del mundo y a través de variadas técnicas estadísticas tales como las de análisis factorial confirmatorio AFC y análisis de ecuaciones estructurales SEM.

En América Latina, se pudo confirmar cómo el modelo mayormente aplicado ha sido el Modelo Servqual confirmando su potencia conceptual y progresivamente confirmado dada su característica flexibilidad interna y adaptabilidad externa de las dimensiones iniciales del modelo, las cuales pudieron ser perfectamente ajustadas a diversos contextos de servicio y organizacionales.

Los hallazgos de la recopilación bibliográfica del presente estudio permite confirmar que el modelo Servqual puede resultar una medida efectiva de la calidad de servicio. Sin embargo, dado la variabilidad de los ajustes de las dimensiones encontradas en diferentes estudios, la estructura y número de factores del modelo y sus respectivas escalas, ha sido sistemáticamente ratificada en las diversas realidades dónde ha sido aplicado.

\section{Referencias}

Abuosi, A. A., \& Atinga, R. A. (2013). Service quality in healthcare institutions: establishing the gaps for policy action. International journal of health care quality assurance, 26(5), 481-492.
Adepoju, O. O., Opafunso, Z., \& Ajayi, M. (2018). Primary Health Care in South West Nigeria: Evaluating service quality and patients' satisfaction. African Journal of Science, Technology, Innovation and Development, 10(1), 13-19. Aghamolaei, T., Eftekhaari, T. E., Rafati, S., Kahnouji, K., Ahangari, S., Shahrzad, M. E., ... \& Hoseini, S. H. (2014). Service quality assessment of a referral hospital in Southern Iran with SERVQUAL technique: patients' perspective. BMC health services research, 14(1), 322. $\begin{array}{ccccc}\begin{array}{c}\text { Aguayo, } \\ \text { Vergara }\end{array}{ }_{\text {Editor }} \text { 1993. Ej } & \text { Método A. Buenos } & \text { Deming. } & \text { Javier } \\ \text { Aires. Año } 1993 .\end{array}$ -Borie, H. M. \& SheikhDamanhouri, A. M.(2013). Patients'satisfaction of service quality in Saudi hospitals: a SERVQUAL analysis. of service quality in Saudi hospitals. a SERVQUAL analysis.

Almeida, R. S., Nogueira, L. A., \& Bourliataux-Lajoine, S. (2013). Analysis of the user satisfaction level in a public physical therapy service. Brazilian journal of physical therapy, 17(4), 328-335.

Al-Momani, M. M. (2015). Gap analysis between perceptions and expectations of medical-surgical patients in a public hospital in Saudi Arabia. Medical Principles and Practice, 25(1), 79-84.

Berelson,B.ySteiner,G.1964.Human Behavior; AnInventory of Scientific Findings, (New Cork: Harcout Brace Jovanovich, 1964) p.88

Cabello Emilio, Chirinos Jesús L, 2012. Validation And Applicability of Servqual Modified Survey To Measure External User Satisfaction In Health Services. Revmedhered. 2012; Pág. 23.

Cabero, J. (2002) Utilización de recursos y medios en los procesos de enseñanza-aprendizaje. Universidad de Jaén, 55-76.

Cabero, J. (2004) No todo es Internet: Los medios audiovisuales e informáticos como recursos didácticos. Universidad de Sevilla.

Cengiz, H., \& Fidan, Y. (2017). Comparing alternative service quality scales: An investigation using confirmatory factor analysis in a health care setting. Services Marketing Quarterly, 38(1), 15-22.

Chassin, M. R., \& Galvin, R. W. (1998). The urgent need to improve health care quality: Institute of Medicine National Roundtable on Health Care Quality. Jama, 280(11), 1000-1005.

Chen, Y., Spohrer, J., \& Lelescu, A. (2008). Three factors to sustainable service system excellence: A case study of service systems. Services Computing, 2008 .... Retrieved from http://ieeexplore.ieee.org/abstract/document/4578516/

Chroeder, R. 1992. Administraci6n De Operaciones. Tercera Edición. Editorial Mcgraw-Hill. México.

Costa, I. A., Trevizan, M., de Godoy, S., Nogueira, P., Arena, C.., \& Bis, C. (2018). Expectations and perceptions of clients concerning the quality of care provided at a Brazilian concerning arilian

Cronin Jr, J. J., \& Taylor, S. A. (1992). Measuring service quality: a reexamination and extension. The journal of marketing, 55-68.

Cronin Jr, J. J., Baker, T. L., \& Hawes, J. M. (1994). An assessment of the role performance measurement of power-dependency in marketing channels. Journal of Business Research, 30(3), 201-210.

Cubero, R. (2005) Perspectivas Constructivistas. Editorial GRAÓ, Barcelona. 2005. Extracto Págs. 132 - 135. Díaz Rocío, 2005. La Calidad Percibida En La Sanidad Pública, Rev. Calidad Asistencial. 2005; 20 (1):35-42).

Dolors Setó Pamies, 2014. De La Calidad De Servicio A La Fidelidad Del Cliente Editorial Esic, Deposito Legal: M. 558-2014 Pág. 16. 
Donabedian, A. (1966). Evaluating the quality of medial care. The Milbank memorial fund quarterly, 44(3), 166-206. Edvardsson, B., \& Tronvoll, B. (2013) A new conceptualization of service innovation grounded in S-D logic and service systems. International Journal of Quality and Service Sciences, 5(1), 19-31. https://doi.org/10.1108/17566691311316220 Edvardsson, B., Skålén, P., \& Tronvoll, B. (2012). Service systems as a foundation for resource integration and value co-creation. Review of Marketing Research, 9, 79-
https://doi.org/10.1108/S1548-6435(2012)oooooogoo8 126. https://doi.org/10.1108/S1548-6435(2012)oooooogoo8

Fan, L. H., Gao, L., Liu, X., Zhao, S. H., Mu, H. T., Li, Z., ... \& Lou, F. G. (2017). Patients perceptions of service quality in China: An

Forero, D. E., \& Gómez, A. (2017). Comparison of measurement models based on expectations and perceived performance for the satisfaction study in health services. suma psicológica, 24(2), 87-96.

Frow, P., \& Payne, A. (2011). A stakeholder perspective of the value proposition concept. European Journal of Marketing, $45(1 / 2), \quad 223-240 . \quad$ https://doi.org/10.1108/03090561111095676

Galván, H. R., Moctezuma, J. M., Dolci, G. F., \& López, en los servicios de salud. Revista Conamed $17(4)$

Carrard, F. \& Narayan, H. (2013). Assessing obstetric patient experience: a SERVQUAL questionnaire. International journal of health care quality assurance, 26(7), 582-592.

Gheorghe, I. R., Gheorghe, C. M., \& Purcărea, V. L. (2018). Measuring the perceived quality of ophthalmology services in private organizations. A marketing perspective. Romanian journal of ophthalmology, 62(1), 54.

Grönroos, C. (1984). A service quality model and its marketing implications. European Journal of marketing, 18(4), 36-44. Gronroos, C. (1988). Service quality: The six criteria of good perceived service. Review of business, 9(3), 10.

Hernández Sampieri Roberto, Fernández Collado Carlos, Baptista Lucio Pilar, 2016. Metodología De La Investigacion. cuarta edición. Editorial Mc Graw Hill año 2016 pág. 435.
Horovitz, J. 1991. La Calidad Del Servicio. Editorial Mcgraw-Hill. Madrid. España 1991; Pp. 2-3. Horovitz,J.1991. La Calidad del Servicio. Editorial McGrawHill,Madrid,España Kotler,P.1996.Dirección de Mercadotecnia: Análisis, Planeación, Implementación y Control.

Jadika, K., Khoo, C., \& Na, J. (2011). Literature Review Writing: A Study of Information Selection from Cited Papers. In Asia Pacific Conference Information \& Information Education \& Practice (pp. 467-477). Library \& Information Education \& Practice (pp. 467-477).
Jandavath, R. K. N., \& Byram, A. (2016). Healthcare service quality effect on patient satisfaction and behavioural intentions effect on patient satisfaction and behavioural intentions
in corporate hospitals in India. International Journal of Pharmaceutical and Healthcare Marketing, 10(1), 48-74.

Javed, S. A., \& Ilyas, F. (2018b). Service Quality and Satisfaction in Healthcare Sector of Pakistan-The Patients' Expectations. International Journal of Health Care Quality Assurance, (just-accepted), oo-oo,

Javed, S. A., Liu, S., Mahmoudi, A., \& Nawaz, M. (2018a). Patients' satisfactionandpublicandprivatesectors'healthcareservicequality in Pakistan: Application of grey decision analysis approaches. The International journal of health planning and management.

Kalaja, R., Myshketa, R., \& Scalera, F. (2016). Service quality assessment in health care sector: the case of Durres public hospital. Procedia-Social and Behavioral Sciences, 235, 557-565.

Khamis, K., \& Njau, B. (2014). Patients' level of satisfaction on quality of health care at Mwananyamala hospital in Dar es Salaam, Tanzania. BMC health services research, 14(1), 400. , M., Saha, S., Singh Arya, J., Choubey, G., Chosh, A., Deb Das, K., ... \& Ali, S. (2015). Patient evaluation of
service quality in government homeopathic hospitals service quality in government homeopathic hospitals
in $\mathrm{W}$ est $\mathrm{B}$ engal, I ndia: a cross-sectional survey. Focus on alternative and complementary therapies, 20(1), 23-31. on alternative and complementary therapies, 20(1), 23-31.
(2001). $8^{\circ}$
Lam, $^{\circ}$ S. S. (1997). SERVQUAL: A tool for measuring patients' opinions of hospital service quality in Hong Kong. Total Quality Management, 8(4), 145-152.

Li, M., Lowrie, D. B., Huang, C. Y., Lu, X. C., Zhu, Y. C., Wu, X. H., ... \& Zhao, P. (2015). Evaluating patients' perception of service quality at hospitals in nine Chinese cities by use of the ServOual scale. Asian Pacific Journal of Tropical Biomedicine, 5(6), 497-504.

Likert Ra, 1932. A Technique For Development of Attitude Scales. Archives of Psychology 1932; 140:44-53. Losada Otariola M, Rodriguez Orejuela Agusto, 2007. Calidad De Servicio En Salud: Una Revisión A La Literatura Desde La Perspectiva Del Marketing.Cuad.Adm.Bogota (Colombia),10(34):237-258, Julio-Diciembre De 2007.

Mahía, R, 2002. Redes de Información y Análisis de Datos. Guía de Exposición. Disponible: http:// documentos/factorial.pdf Leído 4 de Noviembre 2013.

Manchado Garabito, R., Tamames Gómez, S., López González, M., Mohedano Macías, L., \& Veiga de Cabo, J. (2009). Revisiones sistemáticas exploratorias. Medicina y seguridad del trabajo, 55(216), 12-19. Marqués, P. (2008) Didáctica. Los procesos de enseñanza y aprendizaje. La motivación. Departamento pedagogía aplicada, Facultad de Educación, Universidad de Barcelona, Barcelona, España, 2008.

Mata Miranda Pilar, Reynoso Arizmendi Francisco, Salazar Valadéz Alberto, 2006. Conceptos básicos de estadística descriptiva útiles para el médico. RevHospGral Dr. M Gea González Vol 7, No.1 Enero-Abril 2006 Págs. 42-46.

Meyer, C., \& Schwager, A. (2007). Understanding Customer Experience. Harvard Business Review, 85(2), 116 124. https://doi.org/10.1108/00242539410067746

Miranda, J. J., Rosales-Mayor, E., Quistberg, D. A., Paca-Palao, A., Gianella, C., Perel, P., ... \& PIAT Working Group. (2013). Patient perspectives on the promptness and quality of care of road traffic incident victims in Peru: a crosssectional, active surveillance study. FroooResearch, 2.

Musa-Juroš, K., Mijoč, J., Horvat, J., Ilakovac, V., Marković, S., \& Racz, A. (2018). Measuring Healthcare Quality( de Atención Primaria de salud de la ciudad de Talca. Facultad de Ciencias Empresariales. Universidad de Talca.

Nadi, A., Shojaee, J., Abedi, G., Siamian, H., Abedini, E., \& Rostami, F. (2016). Patients' expectations and perceptions of service quality in the selected hospitals. Medical Archives, 70(2), 135-139.

Numpaque-Pacabaque, A., \& Rocha-Buelvas, A. (2016). Modelos SERVQUAL y SERVQHOS para la evaluación de calidad de los servicios de salud. Revista dela Facultad deMedicina, 64(4), 715-720.

Oliva, C. Y Hidalgo, C. 2004. Satisfacción Usuaria: Un Indicador De Calidad Del Modelo De Salud Familiar, Evaluada En Un Programa De Atención De Familias En Riesgo Biopsicosocial, En La Atención Primaria. Psykhe, Nov. 2004, Vol.13, No.2, P.173 186.

Oliver, R. L. (1981). Measurement and evaluation of satisfaction processes in retail settings. Journal of Retailing, 57(3), 25-48. Oliver, R. L. (1993). Cognitive, affective, and attribute bases of the satisfaction response. Journal of consumer research, 20(3), 418-430.

Oliver, R.L. (1980). A cognitive model of the antecedents and consequences of satisfaction decisions. Oliver, R L. (1981). "MeasurementandEvaluationofSatisfaction 46o-469. in Retail Settings", Journal of Retailing, vol. $57, \mathrm{n}^{\circ} 3$, pág. 25-48.

Oliver, R.L. (1993): "Cognitive, affective and attribute bases of the satisfaction response". Journal of Consumer Research, vol. 20 (Diciembre), pág. 418-430. Oliver, R.L. (1997): Satisfaction: A Behavioral Perspective on the Consumer. New York: The McGrawHill Companies, Inc. oliver, R.L. (1999): "Whence consumer loyalty?". Journal of Marketing, vol. 63 (special issue), pág. "33- 44. ver, R.L. y Desarbo, W.S. (1988): "Response of Consumer Research, vol. 14 (Marzo), pág. 495-507. Owusu Kwateng, K., Lumor, R., \& Acheampong, F. ${ }^{4}$ O (2017). Service quality in public and private hospitals: A comparative study on patient satisfaction. International Journal of Healthcare Management, 1-8.

Parasuraman A, Zeithaml V, Berry L, 1988. Servqual: A Multiple-Item Scale For Measuring Consumer Perceptions of Service Quality. J Retailing.1988; 6:12-37. arasuraman, A., Zeithaml, V. A., \& Berry, L. L. (1985). A conceptual model of service quality and its implications for future research. the Journal of Marketing, 41-50.

Parasuraman, A., Zeithaml, V. A., \& Berry, L. L. (1988). Servqual: A multiple-item scale for measuring consumer perc. Journal of retailing, 64(1), 12.

Parasuraman, A., Zeithaml, V.A. And Berry, L.L, 1991. "Refinement And Reassessment of The Servqual arasuraman, A.; Zeithaml, V.A. Y Berry, L.L, 1985. "A Conceptual Model of Service Quality And Its Implications For Future Research". Journal Of Marketing, Vol. 49, Pp. 41-50. Pautasso, M. (2013). Ten Simple Rules for Writing a Literature Review. PLoS Computational Biology, 9(7), 7-10. https://doi.org/10.1371/journal.pcbi.1003149

Purcărea, V. L., Gheorghe, I. R., \& Petrescu, C. M. (2013). The assessment of perceived service quality of public
health care services in Romania using the SERVQUAL scale. Procedia Economics and Finance, 6, 573-585.

Rocha, L. R. M., Veiga, D. F., e Oliveira, P. R., Song, E. H., \& Ferreira, L. M. (2013). Health service quality scale: Brazilian Portuguese translation, reliability
and validity. BMC health services research, 13(1), 24. Ruiz, C, 1998. Instrumentos de Investigación Educativa. $\quad$ Segunda Edición. Ediciones CIDEG. Sanfurgo, Claudio, David Olivares (2002). "Determinación del Nivel de Calidad Percibido por los Usuarios de los Servicios de Urgencia, Consultorio Adosado y Pensionado del Hospital de San Fernando". Memoria de Grado para optar al Titulo de Ingeniero Comercial Mención Administración. Universidad de Talca. Schroeder,R.1992. Administración de Operaciones. Tercera Edición.Editorial McGraw-Hill.México

Suter, G. W. (2013). Review papers are important and worth writing. Environmental Toxicology and Chemistry,

32(9), $1929-1930$.
Taylor, S. A. (1993). The roles of service quality, consumer satisfaction, and value in Quinn's (1992) paradigm of services. Journal of Marketing Theory and Practice, 2(1), 14-26.

Teshnizi, S. H., Aghamolaei, T., Kahnouji, K., Teshnizi, S. M. H., \& Ghani, J. (2018). Assessing quality of health services with the SERVQUAL model in Iran. A systematic review and meta-analysis. International Journal for Quality in Health Care, 3o(2), 82-89.

Valencia, A., Piedrahita, L., Zapata, A., Benjumea, Service Quality Domain: A Bibliometric Analysis. 
Journal of Clinical \& Diagnostic Research, 12(8).

Wolniak Radoslaw, Skotnicka-Zasadzien Bozena, 2011. "The Concept Study of Servqual Method's Gap" Published Online: 27 March 2011 ( S Springer Science + Business Media B.V. 2011. Wysong, P. R., \& Driver, E. (2009). Patients' perceptions of nurses' skill. Critical Care Nurse, 29(4), 24-37. Yousapronpaiboon, K., \& C. Johnson, W. (2013). Measuring hospital out-patient service quality in Thailand. Leadership in Health Services, 26(4), 338-355.

Zapata-Gómez, A. (2014). Application of structural equation and servqual in a health service. Dyna, 81(186), 166-174.

Zeithaml, Valerie, Mary Jo Bitner (2002). Marketing de Servicios: Un enfoque de integración del Zeithaml,V y Bitner,M.2001. Marketing de Servicios. Segunda Edición. $\quad$ Editorial McGraw-Hill. Zhuang, W. (2010). Balancing customer and marketing inputs to maximize the value experience. Zun, A. B., Ibrahim, M. I., \& Hamid, A. A. (2018). Level of Satisfaction on Service Quality Dimensions Based on SERVQUAL Model Among Patients Attending 1 Malaysia Clinic in Kota Bharu, Malaysia. Oman medical journal, 33(5), 416-422. 